\title{
High-Efficiency Surface-Induced Dissociation on a Rhenium Oxide Surface
}

\author{
Shai Dagan and Aviv Amirav \\ School of Chemistry, Sackler Faculty of Exact Sciences, Tel Aviv University, Tel Aviv, Israel
}

We report on the high-efficiency surface-induced dissociation of benzene and cyclohexane polyatomic ions after scattering from a rhenium oxide surface with a kinetic energy of 5-290 $\mathrm{eV}$. Rhenium oxide was prepared by directly heating a rhenium metal foil, under $10^{-5} \mathrm{mbar}$ partial oxygen pressure, at about $1000 \mathrm{~K}$.

Rhenium oxide is characterized by a very high work function of $6.4 \mathrm{eV}$ and thus minimizes ion reneutralization probabilities. The catalytic combustion of surface organic impurities with oxygen ensures good long-term stability.

We found that the surface-induced dissociation ion current is 70 times larger on rhenium oxide than on bare rhenium or stainless steel. Absolute scattered ion yields of about $50 \%$ were measured. The implications of surface-induced dissociation on mass spectrometry in supersonic molecular beams are mentioned. (7 Am Soc Mass Spectrom 1993, 4, 869-873)

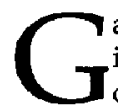
as-phase collision-activated dissociation (CAD) is an established and central technique that contributes to the mass spectrometric identification of organic compounds in tandem mass spectrometry (MS/MS) instruments [1]. Mabud et al. [2] have introduced the technique of surface-induced dissoriation (SID), where the surface replaces the gasphase atoms in mediating the process of CAD. Cooks et al. [3, and references therein] have beautifully demonstrated this technique and many of its features. Recently, SID has attracted considerable interest and attention [4-9]. Basically, SID offers several important advantages over gas-phase CAD: (1) SID is more effective in inducing dissociation, especially in stable, large polyatomic molecules [10]; (2) good control over the internal energy imparted to the scattered ion is available through the control of the ion's incident energy; (3) the internal vibrational energy distribution following surface scattering is narrower than in gasphase CAD. On the other hand, SID suffers from the major disadvantage of ion neutralization on the surfacc, which severely limits its analytical applications. Recently, Cooks and co-workers [11] and Wysocki and co-workers [12] have studied the use of organic monolayer surfaces and found them to be considerably more effective in minimizing surface-induced ion neutralization. In this article we report on our preliminary results of SID of polyatomic molecular ions, utilizing an oxidized rhenium surface.

Rhenium oxide was studied by Fujii et al. [13] who determined that it is the best surface for surface-ioni-

Address reprint requests to Aviv Amirav, School of Chemistry, Sack ler Faculty of Exact Sciences, Tel Aviy University, Ramat Aviv, Tel Aviv 69978, Israel. zation mass spectrometry of organic compounds. Amirav and Danon and co-workers [14-20] have demonstrated that the increase of molecular kinetic energies to the hyperthermal energy range $(1-30 \mathrm{eV})$, dramatically increased the ionization yield of hyperthermal surface ionization (HSI). Several surfaces were investigated, and rhenium oxide was found to be an excellent surface for HSI [18, 21]. Since HSI involves an initial molectule-surface electron transfer, the overall process is a combination of this charge transfer process and a consecutive SID, as demonstrated in cholesterol [21]. It was suggested that thenium oxide could be a very effective surface for the SID of polyatomic ions since (1) it has a very high surface work function of about $6.4 \mathrm{eV}$ [13], and (2) it is formed by heating rhenium foil in an atmosphere of $\sim 10^{-5}$ mbar of oxygen at $1000 \mathrm{~K}$. This suggests that the oxygen and the surface act as a catalytic combustor to continuously clean organic compounds from the surface. This is in contrast to other metal surfaces that, under conventional high-vacuum conditions, become extensively contaminated.

The very high surface work function plays a central role in the very high surface ionization and HSI yield observed. Thus, we anticipate that rhenium oxide will be very effective in promoting high-yield SID [21].

\section{Experimental}

Our apparatus for mass spectrometry in a supersonic molecular beam $[18,22]$ is schematically shown in Figure 1, with its fast gas chromatography inlet [22]. In the experiments presented here, benzene and cyclohexane samples were introduced in a small melting-point tube and vaporized in the injector oven (3). The sample vapor was swept with the helium carrier gas, whose 


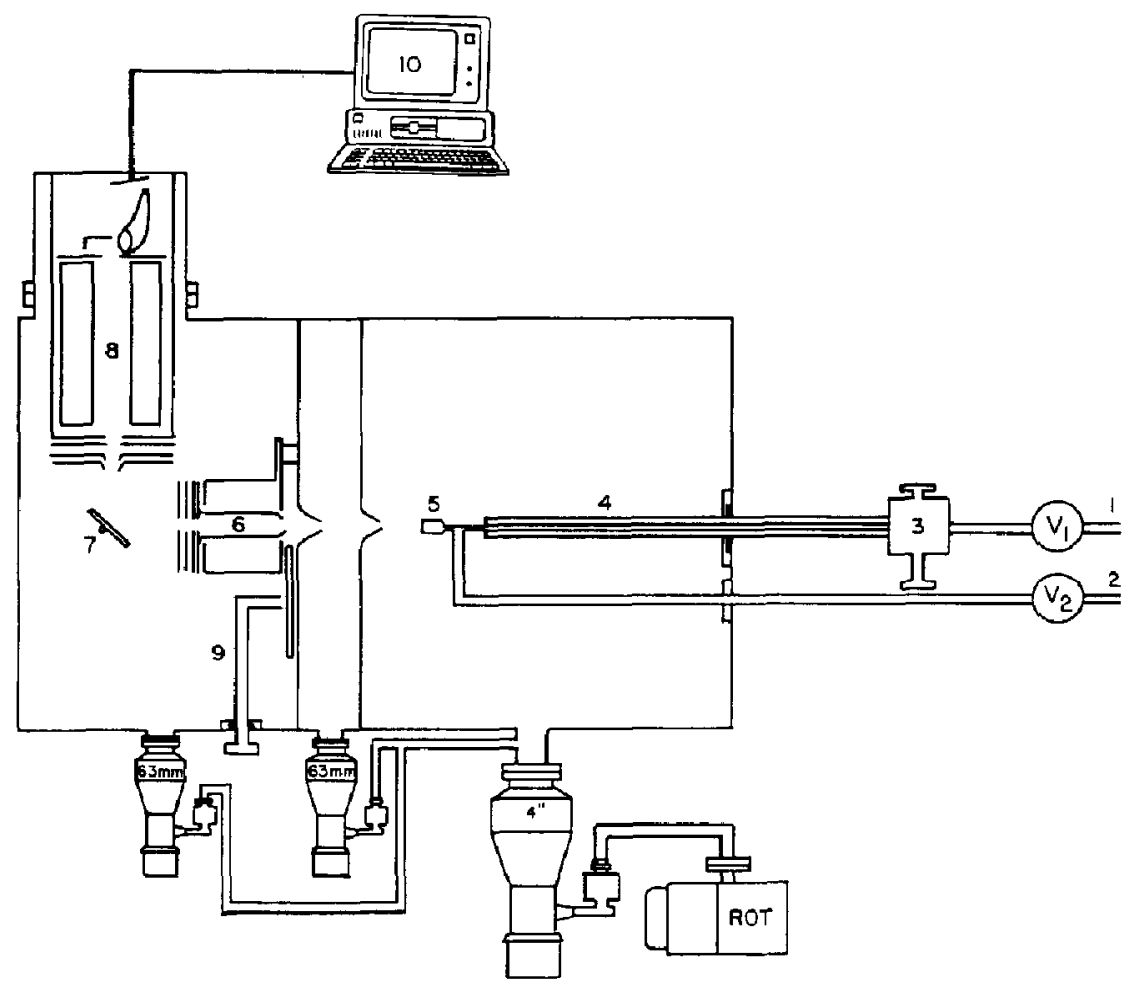

Figure 1. A schematic of the apparatus. The carrier gas ( $\mathrm{He}$ or $\mathrm{H}_{2}$ ) flow is controlled by needle valves (1) and (2). The sample is introduced into the vaporizing oven (3) and flows through the temperature controlled capillary column (4). The gas mixture expands through a supersonic nozzle (5) and is differentially pumped twice. The sample molecules in the beam are ionized at the EI ion source (6) or by the surface (7) (surface and ion deflector are one on top of each other on a vacuum manipulator). They are then mass analyzed by the quadrupole mass spectrometer (8). Alternatively, the EI produced ions are scattered from the thenium oxidre surface (7). The molecular beam can also be square wave modulated by the beam chopper $(y)$. Ihe data are processed and presented on a PC (10).

flow through the column (4) was controlled by needle valve $V_{1}$ and expanded through the supersonic nozzle (5) $[23]$.

The supersonic jet was skimmed and differentially pumped by a 4 -inch diffusion pump (Varian VHS 4) and then by a $63 \mathrm{~mm}$ diffusion pump to create a supersonic molecular beam. The beam entered the mass spectrometer vacuum chamber $(63 \mathrm{~mm}$ diffusion pump) and was then ionized by the fly-through electron-impact (EI) ion source (6) [24]. The electron energy used was $20 \mathrm{eV}$ to reduce the EI fragmentation. The ion fucus plate was positively biased to suppress most of the ions formed from vacuum background molecules as well as low-mass fragment ions that had lower kinetic energy [24]. Thus, this element served as an ion filter, ensuring that the $\mathrm{EI}$ ion source provided a constant flux of predominantly molecular ions of benzene or cyclohexane. The incident benzene molecular ion flux also contained about $7 \%$ relative intensity of $79 \mathrm{Da}$ (molecular ion with $\mathrm{C}^{13}$ isotope) and $77 \mathrm{Da}$, which is due to a hydrugen loss peak. The incident cyclohexane molecular ion flux also contained about $7 \%$ relative intensity each of the 85,69 , and $56 \mathrm{Da}$ spectral peaks. Note that ions formed from molecules in the hyperthermal supersonic molecular beam have additional kinetic energy linearly dependent on their mass due to their aerodynamic acceleration. This kinetic energy was kept low enough to eliminate HSI by using helium as a carrier gas at $150^{\circ} \mathrm{C}$ nozzle temperature. The ions formed in the EI ion source were deflected by $90^{\circ}$ by the positively biased rhenium foil surface ( 7$)$ and mass analyzed by the quadrupole mass spectrometer (QMS) (8) (VG-SXP-600). Alternatively, the rhenium surface was biased at a low positive voltage, below that of the EI ion-source cage, enabling the surface-induced dissociation of the incoming ions. The SID ions were then mass analyzed by the QMS (8) and the data were processed on the PC or presented directly un a chart recorder.

A rhenium foil, $10 \mathrm{~mm} \times 5 \mathrm{~mm} \times 20 \mu \mathrm{m}$, was used as the surface for the SID and HSI (Goodfellow $99.99 \%$ pure). The foil was initially rinsed in organic solvents 
and then cleaned in $10 \%$ nitric acid, followed by brief treatment with $\mathrm{HCl}$. Finally, it was rinsed with doubly distilled water. The foil had to be further cleaned in vacuum by about 20 cycles of heating at $4 \times 10^{-5}$ mbar partial oxygen pressure to about $1700 \mathrm{~K}$, followed by annealing at $2000 \mathrm{~K}$. Its HSI efficiency at $\sim 1000 \mathrm{~K}$ and $2 \times 10^{-5}$ mbar partial oxygen pressure was then monitored. Direct current heating was achieved with 3-8 A.

Good oxidized rhenium surface is characterized by a low optimal HSI "ion energy" surface biasing voltage in the range of 2-10 V. Initially, the HSI ion yield from an unprepared surface was considerably lower and increased with the biasing voltage up to $300 \mathrm{~V}$. Currently, we do not know the exact stoichiometry of the oxide surface formed. We suspect that it is $\mathrm{ReO}_{2}$, since $\operatorname{Re}_{2} \mathrm{O}_{7}$ and $\mathrm{ReO}_{3}$ are more volatile or less stable.

We have crudely measured the scattered ion energy by observing the quadrupole pole bias-voltage effect on the SID mass spectra. We concluded that the scattered ion energy follows the surface voltage and is increased with the incident ion energy and scattered ion mass. Since it is not affected by the incident ion current, we believe that no charge buildup occurs at the rhenium oxide surface.

Both the rhenium foil and an additional stainless steel (304) foil ( $20 \mu$ thick) were mounted vertically one above the other on an XYZR vacuum manipulator, and oxygen was bled directly onto the rhenium surface to convert it into rhenium oxide with a minimal increase in pressure. When hydrogen was used as the carrier gas, about $2 \times 10^{-5}$ mbar partial oxygen pressure was required because of the reaction of hydrogen with the surface oxygen. When helium was used as the carrier gas for the supersonic molectlar beam, the partial oxygen pressure required for good long-term stability was in the $10^{-6}$ mbar range, and under beam-free UHV base pressure conditions, $10^{-6}$ mbar was sufficient [25]. When the oxygen flow was halted, the obtained SID mass spectrum was initially unchanged (no oxygen gas-phase $C A D$ ), and then the SID cffi ciency was gradually reduced over several minutes, predominantly due to the neutral-beam-induced contamination. When rhenium oxide was heated, it emitted alkali ions $\left(\mathrm{Na}^{+}\right.$and $\mathrm{K}^{+}$) and also induced a degree of thermal surface ionization of some unidentified vacuum background molecules. This background was suppressed either by reducing the surface temperature or by electrostatic filtering with the quadrupole entrance lens based on differences between the relatively high-energy SID ions and those emitted by the surface.

\section{Results}

In Figure 2, we present the relative SID mass spectra of benzene produced by the collision of $50 \mathrm{eV}$ molecular ions on the indicated surfaces. The upper mass spectrum (Figure 2a) was achieved following SID on a

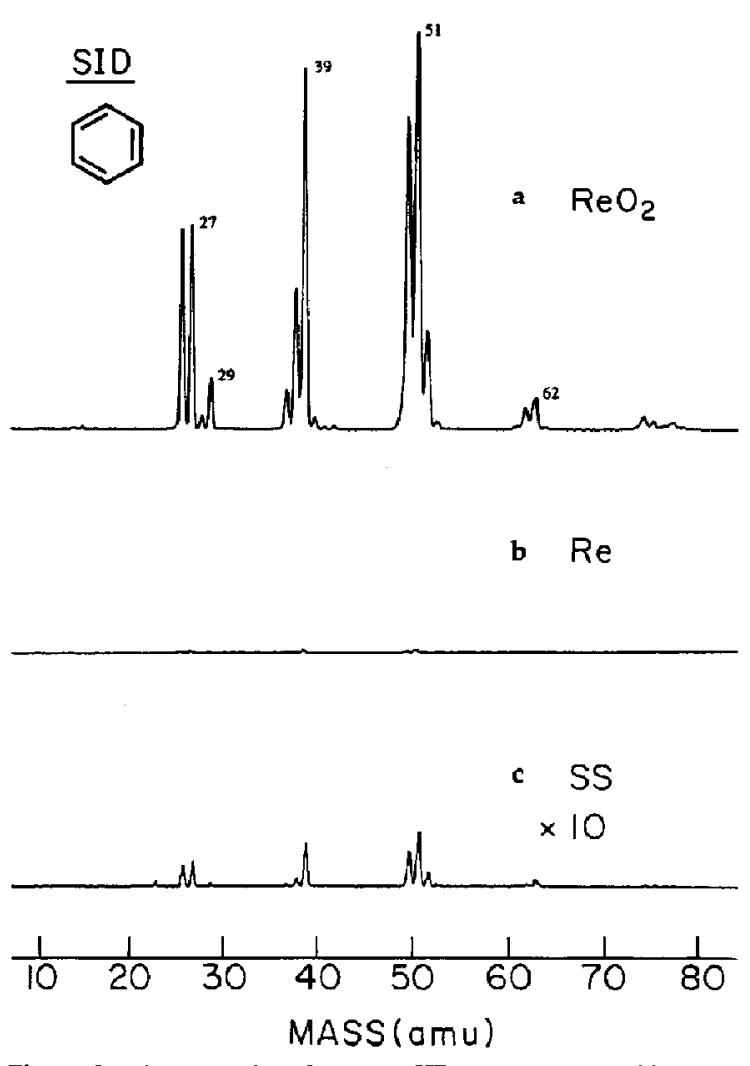

Figure 2. A comparison between SID mass spectra of benzene obtained on a rhenium oxide surface (A), a rhenium surface (B), and a stainless steel 304 surface (C). The benzene ion energy was $50 \mathrm{eV}$.

rhenium oxide surface. It exhibits extensive fragmentation much beyond that exhibited under EI conditions at any electron energy. The resolution is reduced at the higher mass spectral range due to the higher energy of scattered heavy ionic fragments. By introducing pole biasing voltage, the resolution of the higher mass fragments is improved, but under these conditions, the lower-mass fragments are not transmitted. Figure $2 b$ was taken under the identical experimental conditions as Figure 2a but with a bare rhenium foil. The oxygen background was eliminated by closing the oxygen leak valve while the rhenium foil temperature was temporarily increased to $2000 \mathrm{~K}$ to thermally desorb the oxide layer, and then cooling it down to about 800-1000 $\mathrm{K}$ as before. It is clearly observed that the SID fragment ion yield is strongly reduced. By opening the oxygen leak valve, the SID mass spectrum shown in Figure $2 a$ is immediately observed, with a recovery time of a few seconds. In Figure 2c, the SID mass spectrum obtained from stainless steel 304 is shown (magnified 10 times). Note that this SID mass spectrum exhibits about the same intensity as that observed with rhenium, since both are contaminated by organic impurities. We have estimated the SID inte- 
grated yield from the ratio of the integrated mass spectrum intensity to the incoming benzene ion flux. The SID yield on rhenium oxide was about $50 \%$ at 50 $\mathrm{eV}$ incident ion kinetic energy, while on stainless steel it was only $0.7 \%$, and on bare rhenium it was slightly lower. The SID absolute yield depends on the incident ion kinetic energy and at about $30-70 \mathrm{eV}$ it reaches its maximum value.

Note that the actual absolute SID yield can be higher since the fragment ions have high energy and large angular distributions and thus their transmission to and in the QMS is smaller than that of the unscattered, $90^{\circ}$-deflected molecular ion. On the other hand, the dramatic enhancement of the relative SID yield by a factor of 70 seems experimentally clean and easily noticeable, and even higher enhancements are seen at lower ion energies. Note also that on rhenium oxide there is no inherent reason for the ion yield not to exceed $100 \%$ since neutral fragments can also transfer single electrons to the surface as in HSI. Additionally, in Figure 2 we also observe that the SID mass spectra obtained from the stainless-steel surface and the rhenium oxide surface are similar in spite of the large difference in the SID yield. The emerging conclusion is that the ion neutralization process occurs on its incoming trajectory or that the in dissociation process is unimolecular and far from the surface $\left(>10^{-8} \mathrm{~cm}\right)$. The latter conclusion may not be valid for lower energy ions as found in HSI experiments [25], where the ion neutralization probabilities strongly depend on the kinetic energy of the ion or fragment and its ionization potential. A closer examination reveals that the $29 \mathrm{Da}$ spectral peak is unique to SID on rhenium oxide, and it does not appear on rhenium or stainless steel even at higher incident ion energies. We believe that these ions are due to a chemical reaction with the surface [26] that forms the $\mathrm{HCO}^{+}$ions. This conjecture is supported by experiments with deuterated benzene $\left(\mathrm{C}_{6} \mathrm{D}_{6}\right)$.

In Figure 3 we show the SID mass spectra of cyclohexane at the indicated ion energies. It is clearly seen that as with other surfaces, SID on rhenium oxide is a very powerful tool for achieving ion dissociation. Actually, cyclohexane can be complelely dissocialed to very small fragments, and even carbon ions $\left(\mathrm{C}^{+}\right)$are observed in Figure $3 e$.

\section{Discussion}

Currently, the various aspects of the SID of ions produced by EI on rhenium oxide and tungsten oxide are under study, and the results will be reported in a future publication. However, the preliminary results indicate that:

1. The rhenium oxide surface possesses considerable potential for serving as an optimal SID surface. The high yield achieved, combined with the continuous

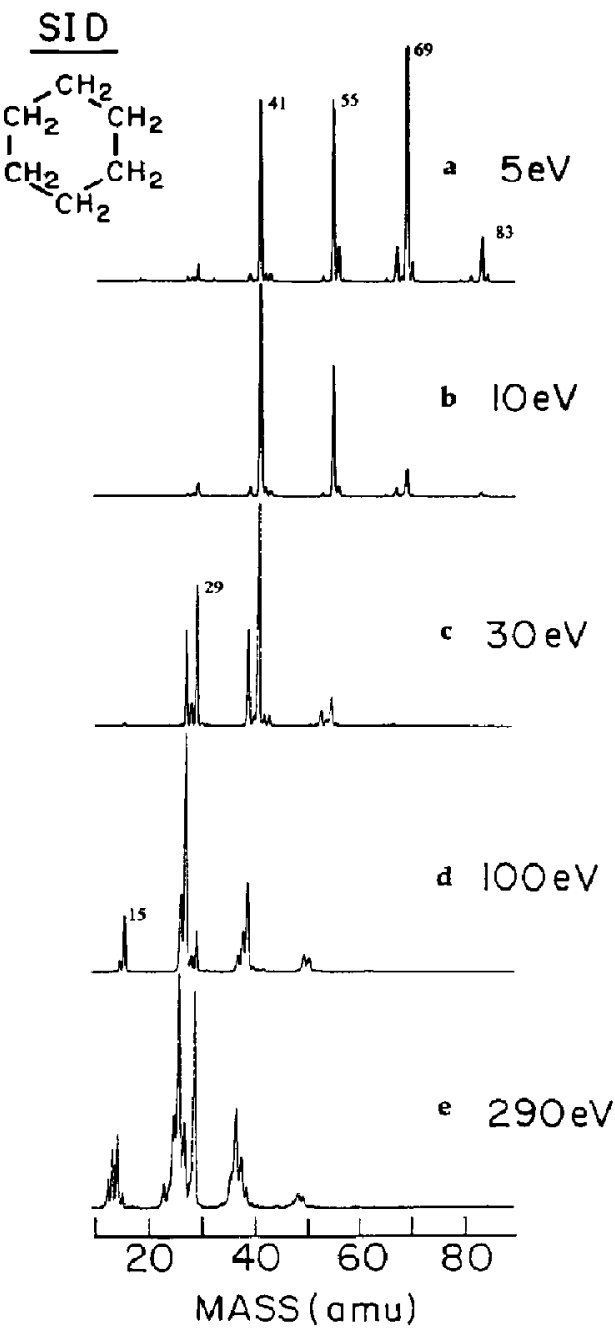

Figure 3. The effect of the kinetic energy of the incident cyclohexane ion (indicated) on the resulting rhenium oxide surface induced dissociation mass spectra. The neutral kinetic energy was about $1.5 \mathrm{eV}$ and this should be added to the indicated electrically given ion energy.

surface cleaning and good long-term stability, makes it competitive with gas-phase CAD.

2. We note that our mass spectrometry system was not modified for these experiments, and it contains the EI ion source and HSI surface in line. Thus, the SID of ions produced by EI adds another dimension of information to our apparatus at no added cost.

3. We anticipate that, in several cases, new fragment ions may appear on the high work function thenium oxide surface following fragmentation at the surface due to reduced ion neutralization [25].

4. Rhenium oxide can also be combined with a gasphase $\mathrm{CAD}$ cell for the hyperthermal surface ion- 
ization of neutral energetic fragments produced in this CAD. This would add another dimension of information to conventional MS/MS.

Finally, in comparing rhenium oxide to organic monolayer surfaces for mass spectrometry applications, it should be mentioned that both surfaces are characterized by good fragment-ion yield. However, the idea of using organic monolayers is based on increasing the ion-metal surface gap to reduce the probability of the neutralization electron transfer process, while with rhenium oxide the surface work function is increased to reduce the surface-ion electron transfer and to increase the probability of neutralsurface electron transfer. The emerging practical differences between these surfaces are:

1. Rhenium oxide requires a continuous flow of oxygen in vacuum, which is generally undesirable.

2. Rhenium oxide is heated to continuously clean itself. The improved long-term stability, however, is combined with a tradeoff of some alkali and thermal surface ionization background that requires filtering. This filtering is more difficult to achieve at low incident ion energies, and a compromise is achieved at intermediate surface temperatures. We believe that under beam-free conditions, such as in MS/MS instruments, unheated rhenium oxide may be used for extended periods of time without extensive contamination.

3. Rhenium oxide surfaces can be used with practically unlimited inn current and energy and show very good long-term stability.

4. SID on rhenium oxide can yield additional informative fragment ions through the HSI of neutral fragments produced in the gas-phase CAD cell or on the surface due to the SID process.

5. Rhenium oxide inherently exists in our apparatus as the HSI surface and thus adds another dimension of information to mass spectrometry in supersonic molecular beams.

\section{Acknowledgment}

This work was supported by a grant from the Wolfson Research Awards, administered by the Israel Academy of Sciences and Humanities.

\section{References}

1. (a) Busch, K. L.; Glish, G. L.; McLuckey, S. A. Mass Spectrometry / Mass Spectrometry, Techniques and Applications of Tandem Mass Spectrometry; VCH: New York, 1988; (b) Burlingame, A.
L.; Baillie, T. A.; Russel, D. H. Anal. Chem. 1992, 64, 467R; (c) McLuckey, S. A. J. Am. Soc. Mass. Spectrom. 1992, 3, 599.

2. Mabud, Md. A.; Dekrey, M. J.; Cooks, R. C. Int. I. Mass Spectrom. Ion Processes 1985, 67, 285.

3. Cooks, R. G.; Ast, T.; Mabud, Md. A. Int. J. Mass Spectrom. Ion Processes 1990, 100, 209.

4. Cole, R. B.; LeMeillour, S.; Tabet, J. C. Anal. Chem. 1992, 64, 365.

5. Wysocki, V. H.; Ding, J. M.; Jones, J. L.; Callahan, J. H.; King, F. L. J. Am. Soc. Mass Spectrom. 1992, 3, 27.

6. Aberth, W. Anal. Chem. 1990, 62, 609.

7. Ijames, C. F.; Wilkins, C. L. Anal. Chem. 1990, 62, 1295.

8. Castro, J. A.; Nuwaysir, L. M.; ljames, C. H.; Wilkins, C. L. Anal. Chem. 1992, 64, 2238.

9. Wright, A. D.; Despeyroux, D.; Jennings, K. R.; Evans, S.; Riddoch, A. Org. Mass. Spectrom. 1992, 27, 525.

10. MeCormack, A. L.; Jones, J. L.; Wysocki, V. H. I. Am. Soc. Mass Spectrom. 1992, 3, 859.

11. (a) Winger, B. E.; Julian, R. K. Jr.; Cooks, R. G.; Chidsey, C. E. D. J. Am. Chem. Soc. 1991, 113, 8967; (b) Morris, M.; Riederer, D. E. Jr.; Winger, B. E.; Cooks, R. G.; Ast. T.; Chidsey, C. E. D. Int. J. Mass Spectrom. Ion Processes 1992, 122, 18.

12. (a) Wysocki, V. H.; Jones, J. L.; Ding, J. M. J. Am. Chem. Soc. 1991, 113, 8969; (b) Somogyi, A.; Kane, T. E.; Ding, J. M.; Wysocki, V. H. I. Am. Chem. Soc. 1993, 115, 527; (c) Wysocki, V. II,; Ding, J. M.; Kane, T. E.; Somogyi, A,; Procetdings of ltte 40th ASMS Conference on Mass Spectrometry and Allied Topics; Washington, DC, 1992; 535.

13. (a) Fujii, T. Int. J. Mass Spectrom. Ion Processes 1989, 87, 51; (b) Fujii, T.; Inagaki, Y.; Mitsutsuka, Y. Int. I. Mass Spectrom. Ion Processes 1993, 124, 45.

14. Amirav, A.; Danon, A. A Method and Apparatus for Producing Ions by Surface Ionization of Energy-Rich Molecules and Atoms, U.S. Patent No. 4845367, 1989; Israel and Great Britain patents approved.

15. (a) Darunl, A.; Anirav, A. J. Chtm. Phys. 1987, 86, 4708; (b) Danon, A.; Amirav, A. Phys. Rev. Lett. 1988, 61, 2961; (c) Danon, A.; Amirav, A. J. Phys. Chem. 1989, 93, 5549; (d) Danon, A.; Amirav, A. Israel J. Chem. 1989, 29, 443; (e) Danon, A.; Amirav, A. Int. I. Mass Spectrom. Ion Processes 1990, 96, 139.

16. Danon, A.; Kolodney, E.; Amirav, A. Surf. Sci. 1988, 193, 132.

17. Amirav, A. Comments Atom Mol. Phys. 1990, 24, 187.

18. Amirav, A. Org. Mass Spectrom. 1991, 26, 1.

19. Danon, $A_{r}$; Vardi, A,; Amirav, A. Phys. Rev. Lett. 1990, 65, 2038.

20. Kuipers, E.; Vardi, A.; Danon, A.; Amirav, A, Phys. Rev. Lett. 1991, 66, 116; Surf. Sci. 1992, 261, 299.

21. Dagan, S.; Danon, A.; Amitav, A. Inf. J. Mass Spectront. Ion Processes 1992, 113, 157.

22. Dagan, 5; Amirav, A. submitted to Anal. Chem.

23. Danon, A.; Amirav, A. Ren. Sri Instrum. 1987, 58, 1724.

24. Amirav, A.; Danon, A. Int. J. Mass Spectrom. Ion Processes $1990,97,107$.

25. Danon, A.; Amirav, A. Int. J. Mass Spectrom. Ion Processes 1993, 125, 63.

26. Ast, T.; Mabud, Md. A.; Cooks, R. G. Int. J. Mass Spectrom. Ion Processes 1988, 82, 131. 